\title{
A Novel Composite-rotating Consensus for Multi-agent System
}

\author{
Gun Li \\ School of Aeronautics and Astronautics \\ University of Electronic Science and Technology of China \\ ChengDu, China \\ ligun@uestc.edu.cn
}

\begin{abstract}
We develop a distributed control policy to achieve composite-rotating consensus around the moving centers whose trajectories form a circle in this paper. It is assumed that the communication topology among agents is undirected network and all agents move on a plane perpendicular to a specified vector in counterclockwise direction. The distributed control protocol is divided into two parts: one part is the local velocity feedback that makes each agent surround the moving centers and the other is the distributed feedback that eliminates the disagreement among the agents. The control protocol is extended to a 3-D space by introduced a rotating matrix. Based on the stable theory of Hurwitz, we verify the stability of the multi-agent system. Finally, Simulation examples are provided to demonstrate the effectiveness of our theoretical results.
\end{abstract}

Keywords-multi-agent system, composite-rotating consensus, 3-D space, moving centers.

\section{INTRODUCTION}

Consensus problem of multi-agent system has received considerable attention by many researchers. Pavone et al. [1] proposed a decentralized control policy for symmetric formations in multi-agent systems that all agents eventually converged to a single point, a circle or a logarithmic spiral pattern. Motivated by the application of autonomous underwater vehicles (AUVs) as mobile sensors to collect oceanographic measurements in formations or patterns that yield maximally information-rich data sets, Sepulchre[2] et al. studied a control strategy to make all multi-agents move in a plane at unit speed. However, these content of researches are confined to 2-D dimension. Motivated by the work of [1], Ren[3] et al. provided the collective motions of a team of vehicles in $3 \mathrm{D}$ by introducing a rotation matrix. In [4], Sonia Hernandez et al. studied decentralized controls to stabilize three-dimensional collective motion of autonomous vehicles and achieved parallel, helical, and circular formations. YANG et al. studied distributed rotating consensus in networks of second-order multi-agent systems using only local position information in three-dimensional (3-D) space in [5]. Lin[6] et al. investigated collective rotating motions of second-order multi-agent system . However, this paper is just in 2-D space. Lin et al. addressed collective rotating motions of second-order multi-agent systems in 3-D space in [7].

In this paper, our main objective is to design a distributed control protocol that has guaranteed all agents achieve composite-rotating consensus in a circular orbit and extended it to 3-D space.

\section{PRELIMINARIES}

\section{A. Graph theory}

For convenience, we suppose the communication in multi-agent system is modeled through an undirected weighted graph $G=(S, e)$ of order $n$ with a set of nodes $S=\left(s_{1}, s_{2}, \cdots, s_{n}\right)$, and a set $e \subseteq S \times S$ of edges, and the edges represent the communication links if node $i$ can get information from node $k$. The set of neighbors of node $s_{i}$ is denoted by $N_{k}=\{k \mid(i, k) \in e, i \neq k\}$. The relation of the communication links can be described by a weighted adjacency matrix $\boldsymbol{A}=\left[a_{i k}\right] \in R^{n \times n}$ with non-negative elements $a_{i k}$ for all $i, k \in I=\{1,2, \cdots, n\}$. The adjacency elements associated with the edges are positive, that is, $e_{i k} \in e \Leftrightarrow a_{i k}>0$. Moreover, we assume $a_{i i}=0$ and $a_{i k}=a_{k i}$ for all $i, k \in I$. A diagonal matrix $\boldsymbol{\Delta}=\operatorname{diag}\left[\boldsymbol{\Delta}_{1}, \boldsymbol{\Delta}_{2}, \ldots, \boldsymbol{\Delta}_{n}\right]$ is a degree matrix of $G$, whose diagonal elements $\boldsymbol{\Delta}_{i}=\sum_{k=1}^{n} a_{i k}$ for all $i=1,2, \cdots, n$. Then, the Laplacian of the undirected graph $G$ is defined as $\boldsymbol{L}=\boldsymbol{\Delta}-\boldsymbol{A}$.

Lemma 1: If an undirected graph $\mathrm{G}$ is connected, then the matrix $L$ is positive.

Lemma 2: Let $k \epsilon R^{3}$ be a unit vector whose coordinate representation in $\Gamma_{1}$ is $k=\left[k_{x}, k_{y}, k_{z}\right]^{T}$, and $\Gamma_{3}$ be a new coordinate system obtained by rotating $\Gamma_{1}$ about the axis $k$ with an angle $\theta$. Then the rotation matrix of $\Gamma_{3}$ with respect to $\Gamma_{1}$ is

$$
\begin{aligned}
& \qquad R_{k}(\theta)=(\cos \theta) I_{3}+(1-\cos \theta) k k^{T}+(\sin \theta) S(k) \\
& \text { where } S\left(k_{. \rightleftharpoons}^{\triangleq}\left[\begin{array}{ccc}
0 & -k_{z} & k_{y} \\
k_{z} & 0 & -k_{x} \\
-k_{y} & k_{x} & 0
\end{array}\right] .\right.
\end{aligned}
$$

\section{B. Transformation of Coordinates}

In this subsection, we introduce some concepts and results of transformation of coordinates. Suppose two coordinates in three space and share a common origin, 
denoted by $\Gamma_{m}(x, y, z)$ and $\Gamma_{n}(\alpha, \beta, \gamma)$. Consider an arbitrary vector $\xi$ expressed in terms of the components along the $x, y, z$ axes and the $\alpha, \beta, \gamma$ axes. Thus we have the equivalent representations:

$$
\xi_{m}=x i_{x}+y i_{y}+z i_{z} \quad \xi_{n}=\alpha i_{\alpha}+\beta i_{\beta}+\gamma i_{\gamma}
$$

where $i_{x}, i_{y}, i_{z} \in \mathbb{R}^{3}$ and $i_{\alpha}, i_{\beta}, i_{\gamma} \in \mathbb{R}^{3}$ are two sets of orthogonal unit vectors parallel to their respective coordinate axes. The subscript of $\xi$ is corresponding to the relevant coordinate system. For convenience, the vector can be written in the manner of matrix:

$$
\xi_{m}=\left[x_{m}, y_{m}, z_{m}\right]^{T} \quad \xi_{n}=\left[\alpha_{n}, \beta_{n}, \gamma_{n}\right]^{T}
$$

The two vectors above can transform mutually by a rotation matrix $R$. Then, we obtain

$$
\Gamma_{n}=R_{n m} \Gamma_{m} \text { or } \Gamma_{m}=R_{n m}^{T} \Gamma_{n}
$$

where $R \in \mathbb{R}^{3 \times 3}$ and it has the property that $R R^{T}=R^{T} R=I_{3}$.

\section{PROBLEM DESCRIPTION}

The distributed system investigated in this paper consists of $n$ autonomous agents. Agent $s_{i}$ can be regard as a node in an undirected graph $G$ and an edge $e \subseteq S \times S$ of graph $G$ corresponds to an information link between agent $s_{i}$ and $s_{k}$. Suppose the dynamics of the ith agent are given by follows:

$$
\dot{r}_{i}=v_{i}, \dot{v}_{i}=u_{i}, i=1,2, \cdots, n
$$

where

$$
r_{i}=\left[r_{i 1}, r_{i 2}, r_{i 3}\right]^{T} \in \mathbb{R}^{3}
$$

$v_{i}=\left[v_{i 1}, v_{i 2}, v_{i 3}\right]^{T} \in \mathbb{R}^{3}$ are respectively the position and speed of agent $s_{i}$ in three dimension and $u_{i} \in \mathbb{R}^{3}$ is the control protocol. Note that all the vectors are represented in an inertial rectangular coordinate system, denoted by $\Gamma_{o}$ in this paper. Due to the application of distributed system in satellite and unmanned aerial vehicle control, etc., composite-rotating consensus of second-order multi-agent system is studied in this paper and extended into three dimension .That is to say ,each agent not only moves around a common axis but also on its own axis.

Definition 1 The multi-agent system (4) achieves composite-rotating consensus if every agent satisfies

$$
\begin{aligned}
& \lim _{t \rightarrow+\infty} i_{\rho}^{T} v_{i}(t)=0 \\
& \lim _{t \rightarrow+\infty}\left[r_{i}(t)-r_{k}(t)\right]=0 \\
& \lim _{t \rightarrow+\infty}\left[v_{i}(t)-v_{k}(t)\right]=0
\end{aligned}
$$

$$
\begin{aligned}
& \lim _{t \rightarrow+\infty}\left[\dot{r}_{i}(t)+\omega_{1}^{-1} R_{n o}^{T} R^{\perp} R_{n o} \dot{v}_{i}(t)-\omega_{2} R_{n o}^{T} R^{\perp} R_{n o}\left(r_{i}(t)\right.\right. \\
& \left.\left.+\omega_{1}^{-1} R_{n o}^{T} R^{\perp} R_{n o} v_{i}(t)\right)\right]=0
\end{aligned}
$$

for any $i, k \in I$, where $i_{\rho} \in \mathbb{R}^{3}$ is a specified vector, $\omega_{1} 、 \omega_{2}$ are positive constants and $\omega_{1} \gg \omega_{2}$,

$$
R^{\perp}=\left[\begin{array}{ccc}
0 & -1 & 0 \\
1 & 0 & 0 \\
0 & 0 & 1
\end{array}\right] .
$$

For convenience in discussion, we assume that all agents move in anticlockwise direction. Besides, we recommend a new rectangular coordinate system $\Gamma_{n}$ whose third coordinate axis is parallel to the specified vector $i_{\rho} \in \mathbb{R}^{3 \times 3}$ Meanwhile, $\Gamma_{o}$ and $\Gamma_{n}$ share the common origin. Thus the rotation matrix that transforms $\Gamma_{o}$ to $\Gamma_{n}$ is denoted as $R_{n o} \in \mathbb{R}^{3 \times 3}$. Here, we do not give any definition of the other two axes for $\Gamma_{n}$ so that the rotation matrix $R_{n o}$ can take different values which have no influence on results.

\section{MAIN RESULTS}

In this section, we investigate the composite- rotating consensus control of the multi-agent system (4) and obtain the following consensus protocol:

$$
u_{i}=u_{i 1}+u_{i 2}
$$

where

$$
\begin{aligned}
& \begin{aligned}
& u_{i 1}=\omega_{1} R_{n o}^{T} \bar{R}^{\perp} R_{n o} v_{i}-\omega_{1} \omega_{2}\left(r_{i}+\omega_{1}^{-1} R_{n o}^{T} \bar{R}^{\perp} R_{n o} v_{i}\right) \\
& \text { and } \\
& u_{i 2}=-\sum_{s_{k} \in N_{i}} a_{i k}\left[v_{i}-v_{k}\right]-\sum_{s_{k} \in N_{i}} a_{i k}\left[\left(r_{i}+\omega_{1}^{-1} R_{n o}^{T} R^{\perp} R_{n o} v_{i}\right)\right. \\
&\left.-\left(r_{k}+\omega_{1}^{-1} R_{n o}^{T} R^{\perp} R_{n o} v_{k}\right)\right], \text { for all } i, k \in I=\{1,2, \cdots, n\}
\end{aligned}
\end{aligned}
$$

and $\bar{R}^{\perp}=\left[\begin{array}{ccc}0 & -1 & 0 \\ 1 & 0 & 0 \\ 0 & 0 & -1\end{array}\right]$. In the protocol (9), $u_{i 1}$ is a local velocity feedback that makes all agents surround a moving point on a plane perpendicular to the vector $i_{\rho}$ and the trajectory of the point is a circle, while $u_{i 2}$ is a distributed state information feedback that eliminates the disagreement dynamics of the multi-agent system.

Let $\xi=\left[r_{1}^{T}, v_{1}^{T}, \cdots, r_{n}^{T}, v_{n}^{T}\right]^{T}$,

And

$$
A=\left[\begin{array}{ll}
0 & I_{3} \\
-\omega_{1} \omega_{2} I_{3} & \left(\omega_{1}-\omega_{2}\right) R_{n o}^{T} \bar{R}^{\perp} R_{n o}
\end{array}\right]
$$

$$
B=\left[\begin{array}{cc}
0 & 0 \\
I_{3} & I_{3}+\omega_{1}^{-1} R_{n o}^{T} R^{\perp} R_{n o}
\end{array}\right]
$$

Then using the protocol (9) for system (4), the closed-loop system can be written in a vector form as 


$$
\dot{\xi}=\left(I_{n} \otimes A-L \otimes B\right) \xi
$$

where $L$ is the Laplacian of the graph $G$.

Lemma3: Denote

$$
\begin{aligned}
& \bar{\xi}=\left(I_{2 n} \otimes R_{n o}\right) \xi=\left[\bar{r}_{11}^{T}, \bar{r}_{12}^{T}, \bar{r}_{13}^{T}, \bar{v}_{11}^{T}, \bar{v}_{12}^{T}, \bar{v}_{13}^{T}, \cdots, \bar{r}_{n 1}^{T}, \bar{r}_{n 2}^{T}, \bar{r}_{n 3}^{T},\right. \\
& \left.\bar{v}_{n 1}^{T}, \bar{v}_{n 2}^{T}, \bar{v}_{n 3}^{T}\right]^{T} \in \square^{6 n}
\end{aligned}
$$

where $\bar{r}_{i j}, \bar{v}_{i j} \in \square$.Then the system (10) can be replaced by

$$
\dot{\bar{\xi}}=\left(I_{n} \otimes \bar{A}-L \otimes \bar{B}\right) \bar{\xi}
$$

Where

$$
\bar{A}=\left[\begin{array}{lc}
0 & I_{3} \\
-\omega_{1} \omega_{2} I_{3} & \left(\omega_{1}-\omega_{2}\right) \bar{R}^{\perp}
\end{array}\right]
$$

and

Further,

$$
\bar{B}=\left[\begin{array}{cc}
0 & 0 \\
I_{3} & I_{3}+\omega_{1}^{-1} R^{\perp}
\end{array}\right] .
$$

$\rho_{1}=\left[\bar{r}_{11}^{T}, \bar{r}_{12}^{T}, \bar{v}_{11}^{T}, \bar{v}_{12}^{T}, \cdots, \bar{r}_{n 1}^{T}, \bar{r}_{n 2}^{T}, \bar{v}_{n 1}^{T}, \bar{v}_{n 2}^{T}\right]^{T} \in \mathbb{R}^{4 n}$

$\rho_{2}=\left[\bar{r}_{13}^{T}, \bar{v}_{13}^{T}, \cdots, \bar{r}_{n 3}^{T}, \bar{v}_{n 3}^{T}\right]^{T} \in \mathbb{R}^{2 n}$.Then the system (11) can be decomposed into the following two systems,

$$
\begin{aligned}
& \dot{\rho}_{1}=\left(I_{n} \otimes C-L \otimes D\right) \rho_{1} \\
& \dot{\rho}_{2}=\left(I_{n} \otimes E-L \otimes F\right) \rho_{2}
\end{aligned}
$$

where

$$
\begin{aligned}
& C=\left[\begin{array}{lccc}
0 & 0 & 1 & 0 \\
0 & 0 & 0 & 1 \\
-\omega_{1} \omega_{2} & 0 & 0 & \omega_{2}-\omega_{1} \\
0 & -\omega_{1} \omega_{2} & \omega_{1}-\omega_{2} & 0
\end{array}\right], \\
& D=\left[\begin{array}{cccc}
0 & 0 & 0 & 0 \\
0 & 0 & 0 & 0 \\
1 & 0 & 1 & -\frac{1}{\omega_{1}} \\
0 & 1 & \frac{1}{\omega_{1}} & 1
\end{array}\right], \\
& E=\left[\begin{array}{ll}
0 & 1 \\
-\omega_{1} \omega_{2} & \omega_{2}-\omega_{1}
\end{array}\right] \text { and } F=\left[\begin{array}{cc}
0 & 0 \\
1 & \frac{1}{\omega_{1}}+1
\end{array}\right] .
\end{aligned}
$$

In fact, the system (12) and (13) are the substitutes for system (11) in the rectangular coordinate System $\Gamma_{n}$.

Lemma 4: All roots of the equation

$$
s^{2}+\left(a-b+\frac{c}{a}+c\right) s+a b+c=0
$$

have negative real parts, where $a>b>0, c>0$.

Proof : Take $s=j \omega$ into the equation (14), where $\omega$ is a positive number. Then Eq. (14) is transformed into the following two equations,

$$
\begin{aligned}
& -\omega^{2}+a b+c=0 \\
& \left(a-b+\frac{c}{a}+c\right) \omega=0
\end{aligned}
$$

Form (16), we obtain $\omega=0$ and substitute it into (15), we get

$$
a b+c=0
$$

It is easy to see that the equation (15) cannot hold when $a>b>0, c>0$. That is, the Eq. (14) has no roots on the imaginary axis. What's more, since $a>b>0, c>0$, the coefficients of Eq. (14) are positive. According to the theory of Hurwitz, all roots of Eq. (14) have negative real parts.

Lemma5: If the graph $G$ is connected, then $\lim _{t \rightarrow+\infty}\left[\bar{r}_{i 3}(t)-\bar{r}_{k 3}(t)\right]=0 \quad$ and $\quad \lim _{t \rightarrow+\infty} \bar{v}_{i 3}(t)=0$ for any $i, k \in I$.That is, all agents finally converge to a plane perpendicular to the vector $i_{\rho}$.

Proof: Consider the system (13). By Lemma 1, there exist an orthogonal matrix $\boldsymbol{W} \in R^{n \times n}$ such that $\boldsymbol{W}^{T} \boldsymbol{L} \boldsymbol{W}=\operatorname{diag}\left\{\lambda_{1}, \lambda_{2}, \ldots, \lambda_{n}\right\}$ with $0<\lambda_{1} \leq \lambda_{2} \leq \cdots \leq \lambda_{n}$.It follows that

$$
\begin{aligned}
& \left(\boldsymbol{W}^{T} \otimes \boldsymbol{I}_{3}\right)\left(\boldsymbol{I}_{\boldsymbol{n}} \otimes \boldsymbol{E}-L \otimes \boldsymbol{F}\right)\left(\boldsymbol{W} \otimes \boldsymbol{I}_{3}\right) \\
= & \operatorname{diag}\left\{\boldsymbol{E}-\lambda_{1} \boldsymbol{F}, \boldsymbol{E}-\lambda_{2} \boldsymbol{F}, \cdots, \boldsymbol{E}-\lambda_{n} \boldsymbol{F}\right\}
\end{aligned}
$$

The characteristic polynomial of each $\boldsymbol{E}-\lambda_{i} \boldsymbol{F}$ is $\operatorname{det}\left(s \boldsymbol{I}-\boldsymbol{E}+\lambda_{i} \boldsymbol{F}\right)=s^{2}+\left(\omega_{1}-\omega_{2}+\frac{\lambda_{i}}{\omega_{1}}+\lambda_{i}\right) s+\omega_{1} \omega_{2}+\lambda_{i}$ $=0, i=1,2, \ldots, n$

Then, according to lemma 4, system (18) is Hurwitz stable. In addition, by simple calculation we can obtain that $\left(\boldsymbol{I}_{n} \otimes \boldsymbol{E}-L \otimes \boldsymbol{F}\right) \times\left(1_{n}^{T} \otimes[1,0]\right)^{T}=0$.Then by linear system theory, we have $\lim _{t \rightarrow+\infty} \bar{v}_{i 3}(t)=0$ and $\lim _{t \rightarrow+\infty}\left[\bar{r}_{i 3}(t)\right.$ $\left.-\bar{r}_{k 3}(t)\right]=0$ for any $i, k \in I$. Thus, we can know all agents finally move on a plane perpendicular to the vector $i_{\rho}$.

Lemma 6: All roots of the equation

$$
\begin{aligned}
& s^{4}+2 c s^{3}+\left[c^{2}+2(a b+c)+\left(a-b-\frac{c}{a}\right)^{2}\right] s^{2} \\
& +2 c(a b+c) s+(a b+c)^{2}=0
\end{aligned}
$$

have negative real parts, where $a>b>0, c>0$.

Proof : Take $s=j \omega$ into the equation (19), where $\omega$ 
is a positive number. Then Eq. (19) is transformed into the following two equations,

$$
\begin{aligned}
& \omega^{4}-\left[c^{2}+2(a b+c)+\left(a-b-\frac{c}{a}\right)^{2}\right] \omega^{2}+(a b+c)^{2}=0 \\
& -2 c \omega^{3}+2 c(a b+c) \omega=0
\end{aligned}
$$

From (21), we have $\omega=0$ or $\omega^{2}=a b+c$. Substituting $\omega=0$ or $\omega^{2}=a b+c$ into (20), we get

$$
(a b+c)^{2}=0 \text { or } c^{2}+\left(a-b-\frac{c}{a}\right)^{2}=0
$$

It's easy to see that the equation (20) cannot hold when $a>b>0, c>0$.That is, the equation (19) has no roots on the imaginary axis. Additionally, with regard to (19), we can get the all coefficients of equation (19) as follows $a_{0}=1$,

$$
\begin{gathered}
a_{1}=2 c, \quad a_{2}=c^{2}+2(a b+c)+\left(a-b-\frac{c}{a}\right)^{2}, \\
a_{3}=2 c(a b+c), a_{4}=(a b+c)^{2}
\end{gathered}
$$

then we can obtain (23) and (24)

$$
\begin{aligned}
& \Delta_{2}=a_{1} a_{2}-a_{0} a_{3}=2 c^{3}+2 c * 2(a b+c) \\
& +2 c\left(a-b-\frac{c}{a}\right)^{2}-2 c(a b+c)>0
\end{aligned}
$$

and

$$
\Delta_{2}>\frac{a_{1}^{2} a_{4}}{a_{3}}=\frac{4 c^{2}(a b+c)^{2}}{2 c(a b+c)}=2 c(a b+c)
$$

Therefore, by the theory of Hurwiz, the system (19) is stable.

Theorem 1: Consider a multi-agent system with a fixed topology that is connected. The multi-agent system (4) with protocol (9) can reach composite- rotating consensus.

Proof : By Lemma 1, we can express $L$ to $0<\lambda_{1} \leq \lambda_{2} \leq \cdots \leq \lambda_{n}$. Then, there exist an orthogonal matrix $\boldsymbol{W} \in R^{n \times n}$ such that $\boldsymbol{W}^{T} \boldsymbol{L} \boldsymbol{W}=\operatorname{diag}\left\{\lambda_{1}, \lambda_{2}, \ldots, \lambda_{n}\right\}$.Then, we have

$$
\begin{aligned}
& \left(\boldsymbol{W}^{T} \otimes \boldsymbol{I}_{2}\right)\left(\boldsymbol{I}_{\boldsymbol{n}} \otimes \boldsymbol{C}-L \otimes \boldsymbol{D}\right)\left(\boldsymbol{W} \otimes \boldsymbol{I}_{2}\right) \\
= & \operatorname{diag}\left\{\boldsymbol{C}-\lambda_{1} \boldsymbol{D}, \boldsymbol{C}-\lambda_{2} \boldsymbol{D}, \cdots, \boldsymbol{C}-\lambda_{n} \boldsymbol{D}\right\}
\end{aligned}
$$

The characteristic polynomial of each $\boldsymbol{C}-\boldsymbol{\lambda}_{i} \boldsymbol{D}$ is

$$
\begin{gathered}
\operatorname{det}\left(s \boldsymbol{I}-\boldsymbol{C}+\lambda_{i} \boldsymbol{D}\right)=s^{4}+2 \lambda_{i} s^{3}+\left[\lambda_{i}^{2}+2\left(\omega_{1} \omega_{2}+\lambda_{i}\right)+\left(\omega_{1}-\omega_{2}\right.\right. \\
\left.\left.-\frac{\lambda_{i}}{\omega_{1}}\right)^{2}\right] s^{2}+2 \lambda_{i}\left(\omega_{1} \omega_{2}+\lambda_{i}\right) s+\left(\omega_{1} \omega_{2}+\lambda_{i}\right)^{2}=0, i=1,2, \ldots, n
\end{gathered}
$$

Then, according to Lemma 6, the system (25) is Hurwitz stable. Besides, by simple calculations, we have $\lim _{t \rightarrow \infty}\left[\dot{r}_{i}(t)+\omega_{1}^{-1} R_{n o}^{T} R^{\perp} R_{n o} \dot{v}_{i}(t)-\omega_{2} R_{n o}^{T} R^{\perp} R_{n o}\left(r_{i}(t)+\omega_{1}^{-1} R_{n o}^{T} R^{\perp} R_{n o}\right.\right.$ $\left.\left.v_{i}(t)\right)\right]=0$ for any $i \in I$.Thus, the multi-agent system (4) with the protocol (9) can achieve composite rotating consensus.

\section{SIMULATION}

In this section, we study the composite-rotating consensus using protocol (9). Suppose that the communication topology of undirected network with four agents is given by Fig.1. The problem of compositerotating consensus is extended to $3-\mathrm{D}$ space by introduced a rotating matrix. The initial condition of system (4) is given as

$$
\begin{aligned}
& {\left[\bar{r}_{11}^{T}, \bar{r}_{12}^{T}, \bar{r}_{13}^{T}, \bar{v}_{11}^{T}, \bar{v}_{12}^{T}, \bar{v}_{13}^{T}, \bar{r}_{21}^{T}, \bar{r}_{22}^{T}, \bar{r}_{23}^{T}, \bar{v}_{21}^{T}, \bar{v}_{22}^{T},\right.} \\
& \left.\bar{v}_{23}^{T}, \bar{r}_{31}^{T}, \bar{r}_{32}^{T}, \bar{r}_{33}^{T}, \bar{v}_{31}^{T}, \bar{v}_{32}^{T}, \bar{v}_{33}^{T}, \bar{r}_{41}^{T}, \bar{r}_{42}^{T}, \bar{r}_{43}^{T}, \bar{v}_{41}^{T}, \bar{v}_{42}^{T}, \bar{v}_{43}^{T}\right]^{T}
\end{aligned}
$$

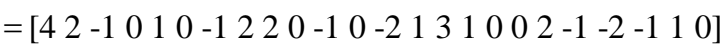

$$
\begin{aligned}
& \text { and } i_{\rho} \text { is taken as }[2 / \sqrt{3}, 2 / \sqrt{3}, 2 / \sqrt{3}]^{T} \text {. Let } \omega_{1}=1
\end{aligned}
$$
and

$$
\omega_{2}=\frac{1}{16} \text {, then we can obtain that, }
$$

$$
C=\left[\begin{array}{lrrc}
0 & 0 & 1 & 0 \\
0 & 0 & 0 & 1 \\
-\frac{1}{16} & 0 & 0 & -\frac{15}{16} \\
0 & -\frac{1}{16} & \frac{15}{16} & 0
\end{array}\right],
$$

$$
D=\left[\begin{array}{rrrr}
0 & 0 & 0 & 0 \\
0 & 0 & 0 & 0 \\
1 & 0 & 1 & -1 \\
0 & 1 & 1 & 1
\end{array}\right], E=\left[\begin{array}{rr}
0 & 1 \\
-\frac{1}{16} & -\frac{15}{16}
\end{array}\right], F=\left[\begin{array}{ll}
0 & 0 \\
1 & 2
\end{array}\right] \text {. }
$$

Clearly, it has been shown in Fig.2 that the moving centers of the four agents reach rotating consensus in a circular orbit. Besides, the multi-agent system finally achieve composite- rotating consensus in a circular orbit around the moving centers and all the agents move in the counterclockwise direction on the plane perpendicular to the vector $i_{\rho}$. Therefore, simulation results have shown the effectiveness of theoretical results.

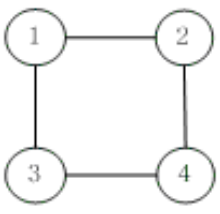

Figure.1 The communication topology of undirected network.

\section{CONCLUSIONS}

The main contribution of this paper is to provide a distributed control policy that allows the agents to achieve composite-rotating consensus in a circular orbit around the moving centers whose trajectories also form a common circle. The control policy is divided into two parts: one part is feedback from itself so that the trajectories of all agents 
finally form a circle and the other is distributed feedback to alleviate divergence among different agents. It's studied in 3-D space by introduced a rotating matrix and all the agents move on a plane perpendicular to the vector $i_{\rho}$. We have proved the effectiveness of the policy proposed in this paper by Hurwitz theory.

\section{REFERENCES}

[1] M. Pavone and E. Frazzoli, Decentralized policies for geometric pattern formation and path coverage[J], ASME Journal of Dynamic Systems, Measurement, and Control, 2007 ,129(5): 633-643.

[2] Sepulchre R, Paley D and Leonard N, Stabilization of Planar Collective Motion With Limited Communication[J], IEEE TRANSACTIONS ON AUTOMATIC CONTROL, 2008,53(3): 706-719.
[3] Wei Ren, Collective Motion From Consensus With Cartesian Coordinate Coupling[J], IEEE TRANSACTIONS ON AUTOMATIC CONTROL,2009,54(6):1330-1335.

[4] Sonia Hernandez and Derek A. Paley, Three-Dimensional Motion Coordination in a Time-Invariant Flowfield[C],Joint 48th IEEE Conference on Decision and Control and 28th Chinese Control Conference,2009:7043-7048.

[5] YANG Tan, WANG Wenyong, HUANG Lishen, Multi-agent Rotating Consensus Using Only Local Position Information in Three-dimensional Space[C]. in Proceedings of the 30th Chinese Control Conference,2011:4787-4791.

[6] P. Lin, Y. Jia, Distributed rotating formation control of multi-agent systems, Systems and Control Letters[J], Systems \& Control Letters, 2010,59(10): 587-595.

[7] Peng Lin ,Kaiyu Qin , Zhongkui Li ,Wei Ren, Collective rotating motions of second-order multi-agent systems in three-dimensional space[J], Systems \& Control Letters,2011,60:365-372.

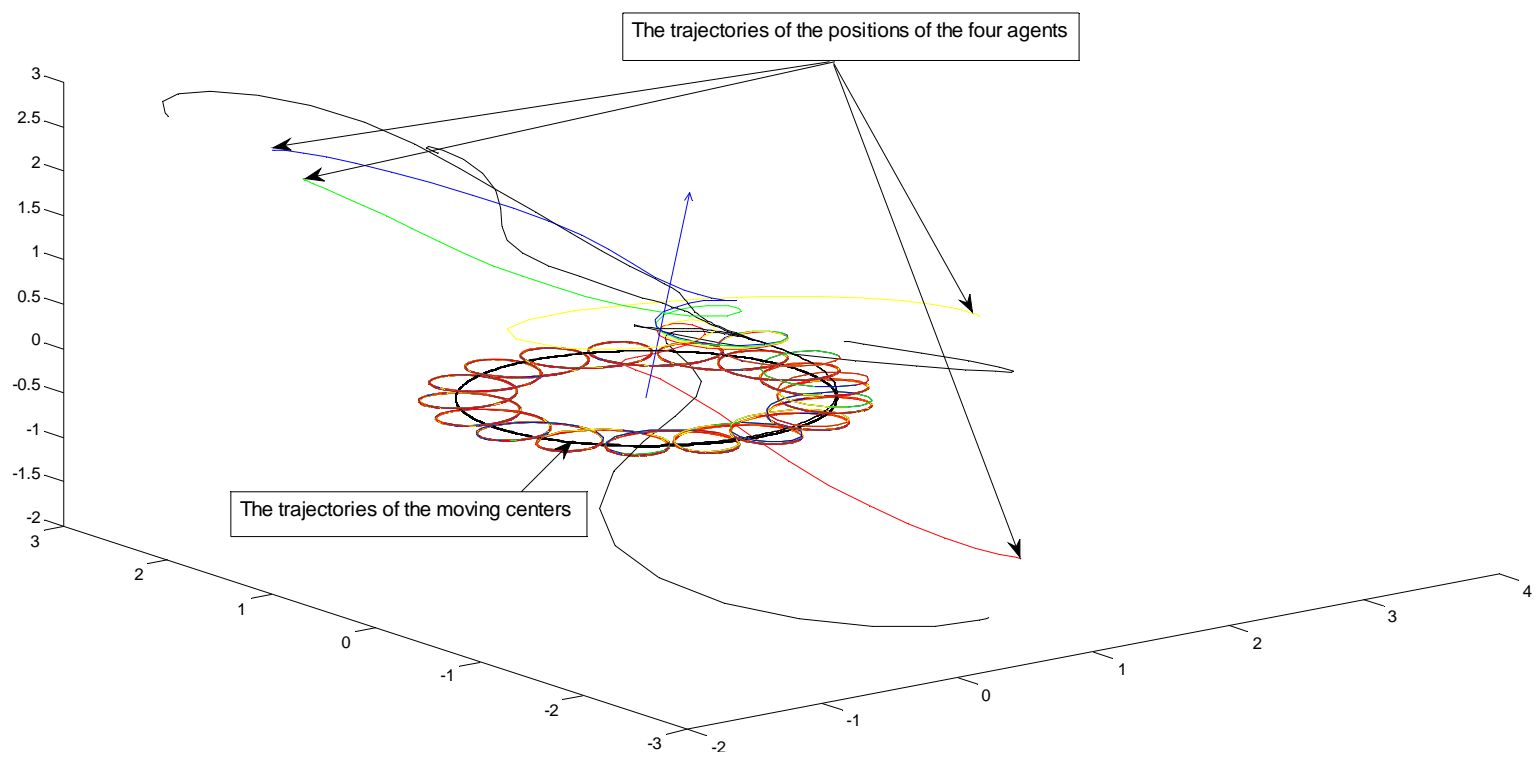

Figure.2 The position trajectories of the multi-agents system. 